\title{
Effect of High-Quality Nursing on Patients with Hemorrhagic Fever with Renal Syndrome
}

\author{
Pule Ren* \\ Huyi District People's Hospital, Xi'an 710300, Shaanxi Province, China \\ *Corresponding author: Pule Ren, 523042502@qq.com
}

Copyright: ( $) 2022$ Author(s). This is an open-access article distributed under the terms of the Creative Commons Attribution License (CC BY 4.0), permitting distribution and reproduction in any medium, provided the original work is cited.

\begin{abstract}
Objective: To explore and analyze the effect of high-quality nursing on patients with epidemic hemorrhagic fever with renal syndrome. Methods: Ninety patients with epidemic hemorrhagic fever with renal syndrome treated in Huyi District People's Hospital from October 2021 to January 2022 were randomly divided into an experimental group and a control group, with 45 cases in each group. The control group received routine nursing and the experimental group received high-quality nursing. The effect of routine nursing and high-quality nursing were compared and analyzed based on the Self-Rating Anxiety Scale (SAS), Self-Rating Depression Scale (SDS), the degree of satisfaction with nursing care, and the patients' quality of life. Results: Before nursing, there was no significant difference in the patients' psychological state between the two groups $(P>0.05)$; after nursing, there was significant difference in the patients' psychological state between the two groups $(P<$ 0.05); the degree of satisfaction with nursing care of the experimental group was significantly higher than that of the control group $(P<0.05)$; comparing the quality of life of the two groups, the quality-of-life score of the control group was significantly lower than that of the experimental group $(P<0.05)$. Conclusion: For patients with epidemic hemorrhagic fever with renal syndrome, high-quality nursing can significantly and effectively improve their psychological state, level of satisfaction with nursing care, and quality of life.
\end{abstract}

Keywords: Hemorrhagic fever with renal syndrome; High-quality nursing; Quality of life; Degree of satisfaction with nursing care

Online publication: March 7, 2022

\section{Introduction}

Epidemic hemorrhagic fever with renal syndrome, also known as epidemic hemorrhagic fever, is a natural focal disease caused by various types of viruses belonging to hantavirus ${ }^{[1]}$, with rats as the source of infection. The main pathological changes include extensive damage to small blood vessels and capillaries in the body. Clinically, patients may present with fever, hypotension or hypotensive shock, congestion, bleeding, and kidney damage. The typical pathological course comprises of the aforementioned five stages, which require active treatment in the hospital when detected early and the use of antiviral drugs under the guidance of doctors ${ }^{[2]}$. In the process of treatment, nursing should be actively given to improve the prognosis and the quality of life of patients. A total of 90 patients with epidemic hemorrhagic fever with renal syndrome treated in Huyi District People's Hospital from October 2021 to January 2022 were selected as the research subjects to determine and analyze the effect of high-quality nursing. 


\section{Materials and methods}

\subsection{General information}

A total of 90 patients with epidemic hemorrhagic fever with renal syndrome who treated in Huyi District People's Hospital from October 2021 to January 2022 were selected and randomly divided into an experimental group $(n=45)$ and a control group $(n=45)$. The control group comprised of 26 male patients and 19 female patients, age ranging from 49 to 71 , with an average age of $58.34 \pm 3.55$. The experimental group comprised of 24 male patients and 21 female patients, age ranging from 47 to 72 , with an average age of $59.90 \pm 5.34$. There was no significant difference in terms of gender, age, and the general data between the two groups $(P>0.05)$. This study was approved by the Ethics Committee of Huyi District People's Hospital, and all the patients had signed the informed consent form prior to their participation.

Inclusion criteria: (1) patients diagnosed with epidemic hemorrhagic fever with renal syndrome via clinical diagnosis ${ }^{[3]}$; (2) patients who are able to communicate normally; (3) patients who have complete clinical treatment data.

Exclusion criteria: (1) cancer patients; (2) patients with diabetes and hypertension; (3) patients with mental disorders.

\subsection{Methods}

\subsubsection{Control group}

The patients in the control group received routine nursing, medication guidance, daily life guidance, and their vital signs were monitored.

\subsubsection{Experimental group}

The patients in the experimental group received high-quality nursing.

(1) Nursing during febrile phase

Patients should be rested in bed in a comfortable and quiet environment to reduce any form of irritation. A high calorie, high vitamin, and digestible diet should be prepared for the patients, and their body temperature should be monitored regularly to look for any changes. Especially for patients with high fever, when their body temperature is too high, they would have to follow the doctor's advice for drug cooling treatment and physical cooling. As this condition involves capillary damage due to toxicity, it is inadvisable to wipe baths with alcohol. The patients' blood pressure should also be measured regularly. In the later stage of the fever, patients tend to sweat a lot and develop blood congestion. Therefore, patients should be encouraged to rehydrate orally more frequently. If necessary, low molecular dextran should be given to prevent shock and protect the kidney.

(2) Nursing during hypotensive phase

The changes of blood pressure should be closely monitored. The patients' blood pressure and pulse should be measured every 30 minutes, and then recorded and reported to the attending doctor in time. It is also important to take note of the speed of fluid replacement. Fluid replacement should be done quickly in the early stage of hypotension through multiple venous channels if necessary. However, for elderly patients, weak patients, and those with heart or renal insufficiency, the speed should be appropriately slowed down, and the dosage should be reduced to prevent the occurrence of pulmonary edema. The patients' 24-hour urine volume should be recorded to detect any early tendency of oliguria. Patients with hypotension should be kept warm and still.

(3) Nursing during oliguric phase

Urine volume, urine specific gravity, presence of fibrin-fibrinogen degradation products in the urine, aggravation of bleeding, and the occurrence of complications should be strictly observed. The intake of water and sodium should be restricted, the speed of rehydration should be controlled, diuretics or 
hemodialysis should be initiated and given according to the doctor's advice, routine blood test should be done, and aseptic procedures should be carried out to prevent infection.

(4) Nursing during polyuric phase

It is necessary to closely observe the patients, follow the doctor's advice, provide adequate fluid treatment to prevent water loss and electrolyte disorders based on the urine measurement of 3,000 ml per day. During this stage, patients are encouraged to have a diet that is easily digested with rich nutrition and high potassium content. For patients with severe anemia, fresh blood can be transfused as appropriate. For patients with daily urine volume of more than 3,000 ml, potassium supplementation should given, mainly orally, and if necessary, it can be given in the form of intravenous drips. Other than potassium, it is important to consider the levels of other electrolytes, such as sodium and calcium, and supplement them if necessary. Hydrochlorothiazide, deoxycorticosterone, pituitrin, indomethacin, and so on can be used for patients with urine volume of more than 5,000 $\mathrm{ml}$ per day. Due to poor immune function, emphasis should be on the prevention of infection. Therefore, indoor air disinfection, oral hygiene, and skin care should be given priority as well.

(5) Nursing during recovery phase

It is necessary to answer the patients' questions, appease the patients, actively dredge their negative emotions, and improve their enthusiasm for treatment. It is also important to ensure a nutritious, high protein, low sugar, and multivitamin diet. The amount of physical activity should be gradually increased over time as the recovery period would generally take about 1-3 months; however, the period of rest should be extended for severe cases.

\subsection{Observation and evaluation}

The satisfaction questionnaire was used to compare the degree of satisfaction with nursing care between the two groups. The scores and their corresponding interpretations are as follows: with a full score of 10 points, 8-10 points denote very satisfied, 5-7 points denote satisfied, and 0-4 points denote dissatisfied. Satisfaction $=($ Very satisfied + Satisfied $) / 45 * 100 \%$

The quality-of-life evaluation scale was used to score the quality of life of the two groups of patients, including material life, psychological, physical, social, and other aspects, with a total score of 70 points for each domain. The higher the score, the better the quality of life.

Self-rating Depression Scale (SDS) and Self-rating Anxiety Scale (SAS) were used to score the psychological state of the patients in the two groups, with a total of 20 items and 4 points for each item. The lower the score, the better the psychological state of the patient.

\subsection{Statistical analysis}

SPSS 27.0 was used to analyze and process the data of the two groups of patients. The measurement data were tested by $t$-test and represented by $\overline{\mathrm{x}} \pm \mathrm{s}$, whereas the count data were tested by $X^{2}$ test and represented by $\%$. $P<0.05$ indicates statistical significance.

\section{Results}

\subsection{Degree of satisfaction with nursing care}

After nursing, the degree of satisfaction of patients in the experimental group with the nursing care was significantly higher than that of the control group. There was significant difference between the two groups $(P<0.05)$ (Table 1). 
Table 1. Comparison of the degree of satisfaction with nursing care between the two groups (n/\%)

\begin{tabular}{cccccc}
\hline Group & Number of cases & Very satisfied & Satisfied & Dissatisfied & Degree of satisfaction \\
\hline Experimental group & 45 & $32(71.11)$ & $12(26.67)$ & $1(2.22)$ & $44(97.78)$ \\
Control group & 45 & $27(60.00)$ & $10(22.22)$ & $8(17.78)$ & $37(82.22)$ \\
$X^{2}$ & & & & & 6.0494 \\
$P$ & & & & & 0.0139 \\
\hline
\end{tabular}

\subsection{Quality of life}

The quality-of-life score of the experimental group was significantly higher than that of the control group $(P<0.05)$, as shown in Table 2.

Table 2. Comparison of quality-of-life scores between the two groups $(\bar{x} \pm s)$

\begin{tabular}{cccccc}
\hline Group & $\mathbf{n}$ & Material life & Social function & Psychological function & Physical function \\
\hline Experimental group & 45 & $57.32 \pm 6.88$ & $61.46 \pm 7.65$ & $59.54 \pm 7.54$ & $60.52 \pm 5.48$ \\
Control group & 45 & $48.78 \pm 7.55$ & $52.34 \pm 7.67$ & $51.12 \pm 6.69$ & $50.83 \pm 5.76$ \\
$t$ & & 5.6085 & 5.6475 & 5.6034 & 8.1761 \\
$P$ & & 0.0000 & 0.0000 & 0.0000 & 0.0000 \\
\hline
\end{tabular}

\subsection{SAS and SDS scores}

Before nursing, there was no significant difference in the psychological state between the two groups $(P>$ 0.05). After nursing, the comparison of the scores that reflect the psychological state of the patients between the two groups showed that the scores of the experimental group were significantly lower than those of the control group $(P<0.05)$, as shown in Table 3 .

Table 3. Comparison of SAS and SDS scores between the two groups before and after intervention $(\overline{\mathrm{x}} \pm \mathrm{s})$

\begin{tabular}{ccccc}
\hline \multirow{2}{*}{ Group } & \multicolumn{2}{c}{ SAS score (point) } & \multicolumn{2}{c}{ SDS score (point) } \\
\cline { 2 - 5 } & Before nursing & After nursing & Before nursing & After nursing \\
\hline Experimental group $(\mathrm{n}=45)$ & $53.52 \pm 3.26$ & $41.67 \pm 2.57$ & $56.45 \pm 1.90$ & $42.76 \pm 1.45$ \\
Control group $(\mathrm{n}=45)$ & $53.44 \pm 3.58$ & $47.42 \pm 3.18$ & $56.45 \pm 1.52$ & $48.67 \pm 1.53$ \\
$t$ & 0.110 & 9.433 & 0.000 & 18.807 \\
$P$ & 0.912 & 0.000 & 1.000 & 0.000 \\
\hline
\end{tabular}

\section{Discussion}

The clinical manifestation of hemorrhagic fever with renal syndrome in the febrile phase is mainly high fever. Many patients will also present with "three pain symptoms and three red symptoms" (headache, lower back pain, and pain in the eye socket; redness over the face, neck, and upper chest). In the hypotensive shock phase, the main symptoms are hypotension and shock. In the oliguric phase, the urine volume significantly reduces, whereas in the polyuric phase, the urine volume significantly increases. In the recovery phase, all symptoms will significantly improve. After treatment, corresponding nursing measures should be taken to improve the treatment effect and quality of life of patients. In this study, after nursing, the degree of satisfaction with nursing care of the experimental group was significantly higher than that of the control group $(P<0.05)$. Before nursing, there was no significant difference in psychological state 
between the two groups $(P>0.05)$, but after nursing, there was significant difference in the psychological state between the two groups $(P<0.05)$. Comparing the quality-of-life scores between the two groups, the scores of the experimental group were significantly higher than those of the control group $(P<0.05)$. This study proves that high-quality nursing is helpful to improving the degree of satisfaction with nursing care among patients with hemorrhagic fever with renal syndrome, their quality of life, and their psychological state.

In conclusion, high-quality nursing is of great significance to patients with epidemic hemorrhagic fever with renal syndrome.

\section{Disclosure statement}

The author declares no conflict of interest.

\section{References}

[1] Chen W, Du H, Wang X, et al., 2021, Levels of Procalcitonin, White Blood Cell and C-reactive Protein in Patients with Hemorrhagic Fever with Renal Syndrome and Their Clinical Significance. Infectious Disease Information, 34(06): 515-518.

[2] Chen H, He W, Zhou X, et al., 2021, Case Report of Hemorrhagic Fever with Renal Syndrome Associated with Dengue Fever in Longhua District, Shenzhen City. Chinese Journal of Endemiology, 40(12): 1021-1023.

[3] Hu Q, Sun W, Hua H, et al., 2021, Antibody Detection and Population Characteristics Analysis of Hemorrhagic Fever with Renal Syndrome Patients at National Monitoring Sites in Heilongjiang Province, 2015-2018. Journal of Tropical Medicine, 21(10): 1343-1345, 1352. 